\title{
The Factorizational Theory of Finite Asymptotic Expansions in the Real Domain: A Survey of the Main Results
}

\author{
Antonio Granata \\ Dipartimento di Matematica e Informatica, Università della Calabria, Cosenza, Italy \\ Email: antonio.granata@unical.it
}

Received 16 October 2014; revised 16 November 2014; accepted 3 December 2014

Copyright (C) 2015 by author and Scientific Research Publishing Inc. This work is licensed under the Creative Commons Attribution International License (CC BY). http://creativecommons.org/licenses/by/4.0/

\section{(c) (i) Open Access}

\section{Abstract}

After studying finite asymptotic expansions in real powers, we have developed a general theory for expansions of type $(*) \quad f(x)=a_{1} \phi_{1}(x)+\cdots+a_{n} \phi_{n}(x)+o\left(\phi_{n}(x)\right), x \rightarrow x_{0}$, where the ordered $n$-tuple $\left(\phi_{1}(x), \cdots, \phi_{n}(x)\right)$ forms an asymptotic scale at $x_{0}$, i.e. $\phi_{1+1}(x)=o\left(\phi_{1}(x)\right)$ as $x \rightarrow x_{0}, 1 \leq i \leq n-1$, and is practically assumed to be an extended complete Chebyshev system on a one-sided neighborhood of $x_{0}$. As in previous papers by the author concerning polynomial, real-power and two-term theory, the locution "factorizational theory" refers to the special approach based on various types of factorizations of a differential operator associated to $\left(\phi_{1}, \cdots, \phi_{n}\right)$. Moreover, the guiding thread of our theory is the property of formal differentiation and we aim at characterizing some $n$-tuples of asymptotic expansions formed by $(*)$ and $n-1$ expansions obtained by formal applications of suitable linear differential operators of orders $1,2, \cdots, n-1$. Some considerations lead to restrict the attention to two sets of operators naturally associated to "canonical factorizations". This gives rise to conjectures whose proofs build an analytic theory of finite asymptotic expansions in the real domain which, though not elementary, parallels the familiar results about Taylor's formula. One of the results states that to each scale of the type under consideration it remains associated an important class of functions (namely that of generalized convex functions) enjoying the property that the expansion $(*)$, if valid, is automatically formally differentiable $n-$ 1 times in two special senses.

\section{Keywords}

Asymptotic Expansions, Formal Differentiation of Asymptotic Expansions, Factorizations of Ordinary Differential Operators, Chebyshev Asymptotic Scales 


\section{Introduction}

In this paper, we give the main results concerning a general analytic theory of asymptotic expansions of type

$$
f(x)=a_{1} \phi_{1}(x)+\cdots+a_{n} \phi_{n}(x)+o\left(\phi_{n}(x)\right), \quad x \rightarrow x_{0} ; n \geq 3,
$$

where

$$
\phi_{1}(x) \gg \phi_{2}(x) \gg \cdots \gg \phi_{n}(x), \quad x \rightarrow x_{o},
$$

and the Hardy notation $\phi(x) \gg \psi(x), x \rightarrow x_{0}$, is alternative to $\psi(x)=o(\phi(x)), x \rightarrow x_{o}$. Though asymptotic expansions are since long a very useful tool in pure and applied mathematics, as far as asymptotic expansions in the real domain are concerned the general theory lacks basic results paralleling, for instance: a) the classical Taylor's formula for polynomial expansions at a point $x_{0} \in \mathbb{R}$; b) the theory of polynomial expansions at $\infty$ systematized in [1]; c) the (not-too-trivial) case $n=2$ thoroughly investigated in [2]. Here, we have in mind characterizations of (1.1) via integro-differential conditions useful for applications unlike the trivial characterization of (1.1) by means of the existence (as finite numbers) of the following $n$ limits defining the coefficients $a_{i}$ :

$$
a_{1}:=\lim _{x \rightarrow x_{0}} f(x) / \phi_{1}(x), \quad a_{i}:=\lim _{x \rightarrow x_{0}} \frac{\left[f(x)-a_{1} \phi_{1}(x)-\cdots-a_{i-1} \phi_{i-1}(x)\right]}{\phi_{i}(x)}, \quad 2 \leq i \leq n,
$$

the $\phi_{i}$ 's being supposed non-vanishing on a deleted neighborhood of $x_{0}$. The three mentioned cases show that a proper approach to a satisfying theory consists in studying (1.1) not by itself but matched to other expansions obtained by formal application of certain differential operators. It is then necessary to make clear what regularity assumptions on the scale are suitable, what types of representations are available for a generic function $f$ in terms of $\left(\phi_{1}, \cdots, \phi_{n}\right)$ and what differential operators are likely to be applicable to (1.1) in order to have a meaningful theory. In $\S 2$, we introduce the concept of Chebyshev asymptotic scale clarifying the relationships between the signs of the two sets of Wronskians $W\left(\phi_{1}, \cdots, \phi_{i}\right)$ and $W\left(\phi_{n}, \cdots, \phi_{n-i}\right)$ which play a decisive role in the sequel: all this matter is indissolubly linked with certain special types of factorizations of disconjugate operators, called canonical factorizations. In $\S 3$, we are led by heuristical considerations to select two sets of differential operators of orders $1,2, \cdots, n-1$ and to formulate conjectures whose proofs are the core of our theory called "the factorizational theory” as in previous papers [1]-[3]. In $\S 4,5,6$, we present the main results; the complete exposition will be published elsewhere but it is available in an electronic archive-arXiv:1406.4321v2 [math.CA], 2014. The main features of this theory are:

(i) It yields applicable analytic characterizations of an expansion (1.1) matched to other asymptotic relations obtained by formal differentiations in suitable senses.

(ii) For each Chebyshev asymptotic scale there are at least two well-defined $(n-1)$-tuples of linear differential operators $\left(L_{1}, \cdots, L_{n-1}\right)$ and $\left(M_{1}, \cdots, M_{n-1}\right)$, of orders $1,2, \cdots, n-1$ respectively, which can be formally applied to (1.1) under suitable integrability conditions. In one of the two circumstances useful representations of the remainders are also available.

(iii) A special family of functions is associated to each Chebyshev asymptotic scale namely that of generalized convex functions, for which the validity of the sole relation (1.1) automatically implies its formal differentiability $(n-1)$ times in the two senses involving the above-mentioned operators $\left(L_{1}, \cdots, L_{n-1}\right)$ and $\left(M_{1}, \cdots, M_{n-1}\right)$.

The introductions in [1] [2] contain other comments.

\section{Notations}

- $f \in A C^{0}(I) \equiv A C(I) \Leftrightarrow f$ is absolutely continuous on each compact subinterval of $I$;

- $f \in A C^{k}(I) \Leftrightarrow f^{(k)} \in A C(I)$;

- For $f \in A C^{k}(I)$ we write $\lim _{x \rightarrow x_{0}} f^{(k+1)}(x)$ meaning that $x$ runs through the points wherein $f^{(k+1)}$ exists as a finite number. Applying L'Hospital's rule in such a context means using Ostrowski's version [4] valid for absolutely continuous functions.

- $\overline{\mathbb{R}}:=\overline{\mathbb{R}} \cup\{ \pm \infty\}$ denotes the extended real line.

- If no ambiguity arises we use the following shorthand notations or similar ones: 


$$
\int_{x}^{x_{0}} f_{1} \int_{t_{1}}^{x_{0}} f_{2} \cdots \int_{t_{n-2}}^{x_{0}} f_{n-1} \int_{t_{n-1}}^{x_{0}} f_{n}(t) \mathrm{d} t:=\int_{x}^{x_{0}} f_{1}\left(t_{1}\right) \mathrm{d} t_{1} \int_{t_{1}}^{x_{0}} f_{2}\left(t_{2}\right) \mathrm{d} t_{2} \cdots \int_{t_{n-2}}^{x_{0}} f_{n-1}\left(t_{n-1}\right) \mathrm{d} t_{n-1} \int_{t_{n-1}}^{x_{0}} f_{n}\left(t_{n}\right) \mathrm{d} t_{n}
$$

wherein each integral $\int_{t}^{x_{0}}$ or, alternatively, $\int_{T}^{t}$ may be a proper or improper integral. A notation such as “ $\int^{\rightarrow x o} f$ convergent" means that $I(\epsilon):=\int_{T}^{x_{0}-\epsilon} f$ exists as a Lebesgue integral for some $T<x_{0}$ and each $\left.\epsilon \in] 0, x_{o}-T\right]$ and that the $\lim _{\epsilon \rightarrow 0^{+}} I(\epsilon)$ exists in $\mathbb{R}$ so defining the improper integral $\int_{T}^{x_{0}} f$.

- The symbol $W\left(u_{1}(x), \cdots, u_{i}(x)\right)$ denotes the Wronskian determinant of the ordered $i$-tuple $\left(u_{1}(x), \cdots, u_{i}(x)\right)$, $(i-1)$ times differentiable at the specified point $x ; W(u(x)):=u(x) . W\left(u_{1}, \cdots, u_{i}\right)$ denotes the Wronskian viewed as the operator $W:\left(u_{1}(x), \cdots, u_{i}(x)\right) \mapsto W\left(u_{1}(x), \cdots, u_{i}(x)\right)$ on a specified interval.

- The acronyms we systematically use: T.A.S. := "Chebyshev asymptotic scale" as in Def. 2.1; C.F. := "canonical factorization" defined in Proposition 2.1-(iv) and (v).

\section{Canonical Factorizations of Disconjugate Operators and Chebyshev Asymptotic Scales}

Our theory is built upon appropriate integral representations stemming from a special structure of the asymptotic scale $\left(\phi_{1}, \cdots, \phi_{n}\right)$ : practically it forms a fundamental system of solutions of a disconjugate equation on a onesided neighborhood of $x_{0}$ such that certain Wronskians do not vanish thereon, a property granted by a result by Levin [5] which justifies our definition of Chebyshev asymptotic scale given below. We preliminarly recall some facts about factorizations of differential operators. In this section $L_{n}, n \geq 2$, denotes a linear ordinary differential operator of type

$$
\begin{gathered}
L_{n} u:=u^{(n)}+\alpha_{n-1}(x) u^{(n-1)}+\cdots+\alpha_{0}(x) u, \quad \forall u \in A C^{n-1}(J), \\
\alpha_{i} \in L_{\text {loc }}^{1}(J), \quad 0 \leq i \leq n-1, J \text { a generic interval of } \mathbb{R},
\end{gathered}
$$

where $L_{\text {loc }}^{1}(J)$ denotes the class of functions Lebesgue-summable on every compact subinterval of $J$. The matters to be discussed depend on the property of disconjugacy and several characterizations involving factorizations are collected in the next proposition where special locutions are defined in the statement itself. For general properties about disconjugacy we refer to the book by Coppel [6] and the paper by Levin [5], and for facts concerning canonical factorizations we refer to the papers by Trench [7] and the author [8] [9].

Proposition 2.1 (Disconjugacy on an open interval via factorizations). For an operator $L_{n}$ of type $(2.1)_{1,2}$, $n \geq 2$, on an open interval ] $a, b[$, bounded or not, the following properties are equivalent:

(i) $L_{n}$ is disconjugate on $] a, b\left[\right.$ in the sense that: every nontrivial solution of $L_{n} u=0$ has at most $(n-1)$ zeros on $] a, b[$ counting multiplicities or, equivalently, has at most $(n-1)$ distinct zeros on $] a, b[$.

(ii) $L_{n} u=0$ has a fundamental system of solutions on $] a, b\left[,\left(u_{1}, \cdots, u_{n}\right)\right.$, satisfying Pólya's $W$-property:

$$
\left.W\left(u_{1}(x), \cdots, u_{i}(x)\right)>0 \quad \forall x \in\right] a, b[, \quad 1 \leq i \leq n,
$$

or equivalently $L_{n} u=0$ has solutions $u_{1}, \cdots, u_{n-1}$ satisfying (2.2) for $1 \leq i \leq n-1$.

(iii) $L_{n}$ has a Pólya-Mammana factorization on $] a, b[$ i.e.

$$
\left.L_{n} u \equiv r_{n}\left[r_{n-1}\left(\cdots\left(r_{1}\left(r_{0}\right)^{\prime}\right)^{\prime} \cdots\right)^{\prime}\right]^{\prime}, \quad \forall u \in A C^{n-1}\right] a, b[,
$$

where the $r_{i}$ 's are suitable functions such that:

$$
\left.r_{i}(x)>0 \quad \forall x \in\right] a, b\left[; \quad r_{i} \in A C^{n-1-i}\right] a, b\left[, \quad 0 \leq i \leq n-1 ; \quad r_{n} \in A C^{0}[a, b] .\right.
$$

(iv) $L_{n}$ has a "canonical factorization (C.F. for short) of type (I) at the endpoint a", i.e. a factorization of type (2.3)-(2.4) with the additional conditions:

$$
\int_{\rightarrow a}\left(1 / r_{i}\right)=+\infty, \quad 1 \leq i \leq n-1,
$$


and a similar "C.F. of type (I) at the endpoint b", i.e. with the $r_{i}$ 's satisfying

$$
\int^{\rightarrow b}\left(1 / r_{i}\right)=+\infty, \quad 1 \leq i \leq n-1 .
$$

(v) For each $c, a<c<b, L_{n}$ has a "C.F. on the interval ]a,c[ which is of type (II) at the endpoint $a$ ", i.e. a factorization (2.3)-(2.4) valid on the interval $] a, c\left[\right.$ and with the $r_{i}$ 's satisfying

$$
\int_{\rightarrow a}\left(1 / r_{i}\right)<+\infty, \quad 1 \leq i \leq n-1,
$$

and $L_{n}$ has a "C.F. on the interval $] c, b[$ which is of type (II) at the endpoint $b$ ", i.e. a factorization (2.3)(2.4) valid on the interval $] c, b\left[\right.$ and with the $r_{i}$ 's satisfying

$$
\int^{\rightarrow b}\left(1 / r_{i}\right)<+\infty, \quad 1 \leq i \leq n-1 .
$$

Remarks. Conditions (2.5) or (2.6) are required to hold for the index $i$ running from 1 to $(n-1)$ : there are no conditions on $r_{0}$ and $r_{n}$. Factorizations in properties (iii)-(iv) are global i.e. valid on the whole given interval $] a, b[$, whereas property (v) claims the existence of local C.F.'s of type (II). A global C.F. of type (I) at a specified endpoint does always exist for a disconjugate operator on an open interval and is "essentially" unique in the sense that the functions $r_{i}$ are determined up to multiplicative constants with product 1 , and this is an historical result by Trench [7] which gave new impetus to the asymptotic theory of ordinary differential equations. The situation is quite different for C.F.'s of type (II). For example the operator $L_{n} \equiv u^{(n)}$ has no global C.F. on $(-\infty,+\infty)$ of type (II) at any of the endpoints for it has only "one" (up to constant factors) PólyaMammana factorization on $(-\infty,+\infty)$, namely

$$
u^{(n)} \equiv\left(\cdots\left(u^{\prime}\right)^{\prime} \cdots\right)^{\prime}
$$

and this is a special contingency characterized in [8] (Thm. 3.3) and in [9] (Thm. 7.1). But the operator $u^{(n)}$ thought of as acting on the space $\left.\left.A C^{n-1}\right] 0,+\infty\right)$, or even on the space $\left.\left.C^{\infty}\right] 0,+\infty\right)$, has infinitely many "essentially" different C.F.'s of type (II), for instance the following ones

$$
u^{(n)} \equiv \frac{1}{(x-c)^{n-1}}\left[(x-c)^{2}\left(\ldots\left((x-c)^{2}\left(\frac{u}{(x-c)^{n-1}}\right)\right)^{\prime} \ldots\right)^{\prime}\right]^{\prime}
$$

which are C.F.'s of type (II) at both the endpoints "0" and " $+\infty$ " whatever the choice of the constant $c<0$. For $c=0$ we get a factorization on $] 0,+\infty)$ which is a C.F. of type (I) at " 0 " and of type (II) at " $+\infty$ "; for $c>0$ we have nonglobal factorizations which are of type (II) at $+\infty$.

C.F.'s are naturally linked to bases of ker $L_{n}$ forming asymptotic scales at one or both endpoints and the following results, due to Levin ([5] § 2), highlight important properties of the Wronskians constructed with an asymptotic scale.

Proposition 2.2 (Wronskians of asymptotic scales and their hierarchies). Let $L_{n}$ be an operator of type $(2.1)_{1,2}$ disconjugate on an open interval $] a, b[$. Then:

(i) Its kernel has some basis $\left(\phi_{1}, \cdots, \phi_{n}\right)$ satisfying:

$$
\left\{\begin{array}{l}
\left.\phi_{i}(x)>0 \text { on some interval }\right] b-\varepsilon, b[, \quad 1 \leq i \leq n ; \\
\phi_{1}(x) \gg \phi_{2}(x) \gg \cdots \gg \phi_{n}(x), \quad x \rightarrow b^{-}
\end{array}\right.
$$

(ii) For each such basis:

$$
\left.W\left(\phi_{n}(x), \phi_{n-1}(x), \cdots, \phi_{i}(x)\right)>0 \text { on the whole interval }\right] a, b[, \quad 1 \leq i \leq n,
$$

noticing the reversed order of the $\phi_{i}$ 's in the Wronskians.

(iii) For any strictly decreasing set of indexes $\left\{i_{1}, \cdots, i_{k}\right\}$, i.e. such that

$$
n \geq i_{1}>i_{2}>\cdots>i_{k} \geq 1, \quad 1 \leq k \leq n-1,
$$


we have:

$$
W\left(\phi_{i_{1}}(x), \cdots, \phi_{i_{k}}(x)\right)>0 \text { on a left deleted neighborhood of } b
$$

and in particular we have the inequalities:

$$
\operatorname{signW}\left(\phi_{1}(x), \cdots, \phi_{i}(x)\right)=(-1)^{i(i-1) / 2} \text { on a left deleted neighborhood of } b, \quad 1 \leq i \leq n .
$$

(iv) For each $k, 1 \leq k \leq n-1$, and for any two distinct and strictly increasing sets of indexes $i_{1}, \cdots, i_{k}$ and $j_{1}, \cdots, j_{k}$ such that $i_{h} \leq j_{h}, 1 \leq h \leq k$, we have

$$
W\left(\phi_{i_{1}}(x), \cdots, \phi_{i_{k}}(x)\right) \gg W\left(\phi_{j_{1}}(x), \cdots, \phi_{j_{k}}(x)\right), \quad x \rightarrow b^{-} .
$$

Notice the ordering of the $\phi_{i}$ 's and the $\phi_{j}$ 's in (2.12): if each $\phi_{i}$ has an order of growth at $b^{-}$greater than that of the corresponding $\phi_{j}$ then the same is true for the Wronskians. In the claim (iii), we have a different ordering of the $\phi_{i}$ 's as this grants the positivity of the Wronskians in (2.10).

To visualize (2.12), we list a few asymptotic scales at $b^{-}$constructed with the Wronskians:

$$
\begin{aligned}
& \left\{\begin{array}{l}
W\left(\phi_{1}, \phi_{2}\right) \gg W\left(\phi_{1}, \phi_{3}\right) \gg \cdots \gg W\left(\phi_{1}, \phi_{n}\right), \\
W\left(\phi_{2}, \phi_{3}\right) \gg W\left(\phi_{2}, \phi_{4}\right) \gg \ldots \gg W\left(\phi_{2}, \phi_{n}\right), \quad x \rightarrow b^{-} ; \\
\cdots \\
W\left(\phi_{n-2}, \phi_{n-1}\right) \gg W\left(\phi_{n-2}, \phi_{n}\right),
\end{array}\right. \\
& W\left(\phi_{1}, \phi_{2}, \phi_{3}\right) \gg W\left(\phi_{1}, \phi_{2}, \phi_{4}\right) \gg \ldots \gg W\left(\phi_{1}, \phi_{2}, \phi_{n}\right), \quad x \rightarrow b^{-} .
\end{aligned}
$$

It is quite important to note the order of the $\phi_{i}$ 's forming the asymptotic scale in (2.7); if we maintain the same ordering in the analogous statement for $x \rightarrow a^{+}$, i.e. $\phi_{1}(x) \gg \phi_{2}(x) \gg \cdots \gg \phi_{n}(x), x \rightarrow a^{+}$, then the Wronskians in (2.8), (2.10) and (2.12) are the same, the essential point being the relative growth-order of the $\phi_{i}$ 's. From the point of view of asymptotic expansions the correct numbering is that adopted by us irrespective of the limiting process. The above results substantiate the following definition of special asymptotic scales wherein we merely fix the neighborhood of $b$ left undefined in Proposition 2.2. From now on, the interval will be denoted as in the two-term theory [2].

Definition (Chebyshev asymptotic scales). The ordered $n$-tuple of real-valued functions $\left(\phi_{1}, \cdots, \phi_{n}\right), n \geq 2$ is termed a "Chebyshev asymptotic scale" (T.A.S. for short) on the half-open interval $\left[T, x_{0}\left[, T \in \mathbb{R}, x_{0} \leq+\infty\right.\right.$, provided the following properties are satisfied:

$$
\begin{gathered}
\phi_{i} \in C^{n-1}\left[T, x_{0}[, \quad 1 \leq i \leq n,\right. \\
\phi_{i}(x) \neq 0 \text { on some left deleted neighborhood of } x_{0}, \quad 1 \leq i \leq n, \\
\phi_{1}(x) \gg \phi_{2}(x) \gg \cdots \gg \phi_{n}(x), \quad x \rightarrow x_{o}^{-}, \\
W\left(\phi_{1}(x), \cdots, \phi_{i}(x)\right) \neq 0 \text { on }\left[T, x_{0}[, \quad 1 \leq i \leq n .\right.
\end{gathered}
$$

Whenever the $\phi_{i}$ 's satisfy the stronger regularity condition

$$
\phi_{i} \in A C^{n-1}\left[T, x_{0}[, \quad 1 \leq i \leq n\right.
$$

they remain associated to the operator:

$$
L_{\phi_{1}, \cdots, \phi_{n}} u:=W\left(\phi_{1}(x), \cdots, \phi_{n}(x), u\right) / W\left(\phi_{1}(x), \cdots, \phi_{n}(x)\right)
$$

which is the unique linear ordinary differential operator of type $(2.1)_{1,2}$, acting on the space $A C^{n-1}\left[T, x_{0}[\right.$ and such that $\operatorname{ker} L_{\phi_{1}, \cdots, \phi_{n}}=\operatorname{span}\left(\phi_{1}, \cdots, \phi_{n}\right)$ 
Remarks. 1. Condition (2.15) is the usual regularity assumption in approximation theory (Chebyshev systems and the like), whereas in matters involving differential equations/inequalities it is natural to assume (2.19). Choosing an half-open interval is a matter of convenience: the point $x_{0}$ involved in the asymptotic relations is characterized as the endpoint not belonging to the interval, possibly $x_{0}=+\infty$, whereas the other endpoint marks off an interval whereon the inequalities involving the Wronskians are satisfied and these in turn allow certain integral representations valid on the whole given interval and essential to our theory.

2. In the definition we have merely supposed the non-vanishingness of various functions instead of specifying their signs as in Proposition 2.2; this avoids restrictions that are immaterial in asymptotic investigations. If the $\phi_{i}$ 's are strictly positive near $x_{0}$ then Levin's theorem provides the exact signs of certain Wronskians.

3. As concrete examples of such asymptotic scales on $[T,+\infty)$ the readers may think of scales whose non-identically zero and infinitely-differentiable functions are represented by linear combinations, products, ratios and compositions of a finite number of powers, exponentials and logarithms. As a rule such functions and their Wronskians have a principal part at $+\infty$ which can be expressed by products of similar functions, hence they do not vanish on a neighborhood of $+\infty$.

When comparing our notations with other authors' results the reader must carefully notice the numbering of the $\phi_{i}$ 's in the asymptotic scale (2.17) and in the Wronskians (2.18).

The concept of Chebyshev asymptotic scale, even under the weak regularity (2.15), admits of useful characterizations which generalize a classical result, ([10]; Ch. XI, Th. 1.2, p. 379) about those special asymptotic scales formed by functions with zeros of increasing multiplicities (namely $0,1, \cdots, n-1$ ) at an endopint of a compact interval; also refer to ([10]; Ch. I) and [11] for locutions and facts about Chebyshev systems. Here we only mention the properties necessary to give meaning to the results stated in this survey.

Proposition 2.3 For $\left(\phi_{1}, \cdots, \phi_{n}\right)$ a T.A.S. on $\left[T, x_{0}[\right.$ we have the inequalities:

$$
\begin{gathered}
\phi_{i}(x) \neq 0 \text { on }\left[T, x_{0}[, \quad 1 \leq i \leq n,\right. \\
W\left(\phi_{n}(x), \phi_{n-1}(x), \cdots, \phi_{i}(x)\right) \neq 0 \text { on }\left[T, x_{0}[, \quad 1, \leq i \leq n,\right. \\
W\left(\phi_{i_{1}}(x), \cdots, \phi_{i_{k}}(x)\right) \neq 0 \text { near } x_{o},
\end{gathered}
$$

for any set of indexes satisfying (2.9) and we also have the hierarchies between the Wronskians stated in Proposition 2.2-(iv) and referred to $x \rightarrow x_{0}^{-}$. Whenever the $\phi_{i}$ 's are strictly positive then all the Wronskians in (2.22) are strictly positive on $\left[T, x_{0}[\right.$, but not necessarily all the Wronskians in (2.18); in this case the inverted $n$-tuple $\left(\phi_{n}, \cdots, \phi_{1}\right)$ is an extended complete Chebyshev system on $\left[T, x_{0}[\right.$. On the contrary, if the given $n$-tuple $\left(\phi_{1}, \cdots, \phi_{n}\right)$ is an extended complete Chebyshev system on $\left[T, x_{0}[\right.$, i.e. all the Wronskians in (2.18) are strictly positive on $\left[T, x_{0}\left[\right.\right.$, then certain integral representations (not reported here) imply that the $\phi_{i}$ 's have alternating signs, namely: $\operatorname{sign} \phi_{i}=(-1)^{i-1}$ on $\left[T, x_{0}[\right.$.

Notice that the converse of the inference “(2.18) $\Rightarrow(2.22)$ ” generally fails as easily checked for the scale:

$$
1 \gg c x+x^{2} \gg x^{2}, \quad x \rightarrow 0^{-}, \quad(c>0)
$$

on the interval $\left(-\infty, 0\left[\right.\right.$, for which we have: $\phi_{1}$ and $W\left(\phi_{1}, \phi_{2}, \phi_{3}\right) \neq 0$ on $(-\infty, 0[$; $W\left(\phi_{1}, \phi_{2}\right) \equiv W\left(1, c x+x^{2}\right)=c+2 x \neq 0$ on $]-c / 2,0[$ but not on $(-\infty, 0[$.

In the next proposition we collect all the facts essential to develop our theory of asymptotic expansions.

Proposition 2.4 (Formulas concerning T.A.S.'s linked to differential operators). Let the ordered $n$-tuple $\left(\phi_{1}, \cdots, \phi_{n}\right)$ satisfy conditions (2.15) to (2.19), hence the operator in (2.20) is disconjugate on the open interval ] $x_{0}[$ and, as such, enjoys the properties in Propositions 2.1 and 2.2. Moreover, as an operator acting on $A C^{n-1}\left[T, x_{0}[\right.$ it has the following further properties:

(i) Define the following $(n+1)$ functions on $\left[T, x_{0}[\right.$ :

$$
\left\{\begin{array}{l}
q_{0}:=1 /\left|\phi_{1}\right| ; \quad q_{1}:=\left(\phi_{1}\right)^{2} /\left|W\left(\phi_{1}, \phi_{2}\right)\right| ; \\
q_{i}:=\left[W\left(\phi_{1}, \cdots, \phi_{i}\right)\right]^{2} /\left|W\left(\phi_{1}, \cdots, \phi_{i-1}\right) \cdot W\left(\phi_{1}, \cdots, \phi_{i+1}\right)\right|, \quad 2 \leq i \leq n-1 ; \\
q_{n}:=\left.\left|q_{0} q_{1} \cdots q_{n-1}\right|\right|^{-1} \equiv\left|W\left(\phi_{1}, \cdots, \phi_{n}\right) / W\left(\phi_{1}, \cdots, \phi_{n-1}\right)\right| .
\end{array}\right.
$$


Then the $q_{i}$ 's satisfy the following regularity conditions:

$$
q_{i}(x)>0 \quad \forall x \in\left[T, x_{0}\left[; \quad q_{i} \in A C^{n-1-i}\left[T, x_{0}\left[, 0 \leq i \leq n-1 ; \quad q_{n} \in A C^{0}\left[T, x_{0}[.\right.\right.\right.\right.\right.
$$

Their reciprocals, left apart $q_{0}$ and $q_{n}$, may be expressed as derivatives of certain ratios

$$
1 / q_{1}(x)=\left|\left(\phi_{2}(x) / \phi_{1}(x)\right)^{\prime}\right| ; \quad 1 / q_{i}(x)=\mid\left[\frac{W\left(\phi_{1}(x), \cdots, \phi_{i-1}(x), \phi_{i+1}(x)\right)}{W\left(\phi_{1}(x), \cdots, \phi_{i-1}(x), \phi_{i}(x)\right)}\right], \quad 2 \leq i \leq n-1,
$$

on the interval $\left[T, x_{0}[\right.$, and

$$
\int_{T}^{x_{0}}\left(1 / q_{i}\right)<+\infty, \quad 1 \leq i \leq n-1 .
$$

Our operator admits of the following factorization on $\left[T, x_{0}[\right.$ :

$$
L_{\phi_{1}, \cdots, \phi_{n}} u \equiv q_{n}\left[q_{n-1}\left(\cdots\left(q_{0} u\right)^{\prime} \cdots\right)^{\prime}\right]^{\prime}
$$

which is a global C.F. of type (II) at both endpoints $T$ and $x_{0}$.

(ii) Our T.A.S. (apart from the signs) admits of the following integral representation in terms of the $q_{i}$ 's:

$$
\left|\phi_{1}(x)\right|=\frac{1}{q_{0}(x)} ; \quad\left|\phi_{i}(x)\right|=\frac{1}{q_{0}(x)} \int_{x}^{x_{0}} \frac{1}{q_{1}} \ldots \int_{t_{i-2}}^{x_{0}} \frac{1}{q_{i-1}}, \quad 2 \leq i \leq n, x \in\left[T, x_{0}[,\right.
$$

hence the $\phi_{i}$ 's, besides being everywhere non-zero on $\left[T, x_{0}[\right.$, have the same order of growth at $T$, namely

$$
\lim _{x \rightarrow T^{+}} \frac{\phi_{i}(x)}{\phi_{j}(x)}=c_{i j} \in \mathbb{R} \backslash\{0\} \quad \forall i \neq j .
$$

In the special case where all the Wronskians in (2.18) are strictly positive, i.e. when $\left(\phi_{1}, \cdots, \phi_{n}\right)$ is an extended complete Chebyshev system on $\left[T, x_{0}\left[\right.\right.$, then the $\phi_{i}$ 's have alternating signs, namely

$$
\operatorname{sign} \phi_{i}=(-1)^{i-1} \text { on }\left[T, x_{0}[\text {. }\right.
$$

(iii) Analogously we define the following $(n+1)$ functions on $\left[T, x_{0}[\right.$ :

$$
\left\{\begin{array}{l}
p_{0}:=1 /\left|\phi_{n}\right| ; \quad p_{1}:=\left(\phi_{n}\right)^{2} / W\left(\phi_{n}, \phi_{n-1}\right) ; \\
p_{i}:=\left[W\left(\phi_{n}, \phi_{n-1}, \cdots, \phi_{n-i+1}\right)\right]^{2}\left|W\left(\phi_{n}, \phi_{n-1}, \cdots, \phi_{n-i+2}\right) \cdot W\left(\phi_{n}, \phi_{n-1}, \cdots, \phi_{n-i}\right)\right|^{-1}, \quad 2 \leq i \leq n-1 ; \\
p_{n}:=\left|p_{0} p_{1} \cdots p_{n-1}\right|^{-1} \equiv\left|W\left(\phi_{n}, \phi_{n-1}, \cdots, \phi_{1}\right) / W\left(\phi_{n}, \phi_{n-1}, \cdots, \phi_{2}\right)\right| .
\end{array}\right.
$$

They satisfy the same regularity conditions on the half-open interval $\left[T, x_{0}\left[\right.\right.$ as the $q_{i}$ 's do in (2.26) and their reciprocals may be expressed as derivatives of the following ratios analogous to those in (2.27):

$$
1 / p_{1}(x)=\left|\left(\phi_{n-1}(x) / \phi_{n}(x)\right)^{\prime}\right| ; \quad 1 / p_{i}(x)=\left\lfloor\left[\frac{W\left(\phi_{n}(x), \cdots, \phi_{n-i+2}(x), \phi_{n-i}(x)\right)}{W\left(\phi_{n}(x), \cdots, \phi_{n-i+2}(x), \phi_{n-i+1}(x)\right)}\right], \quad 2 \leq i \leq n-1,\right.
$$

on the interval $\left[T, x_{0}[\right.$. Moreover:

$$
\int^{x o}\left(1 / p_{i}\right)=+\infty, \quad 1 \leq i \leq n-1 ; \quad \int_{T}\left(1 / p_{i}\right)<+\infty, 1 \leq i \leq n-1 ;
$$

hence, apart from constant factors, the associated factorization

$$
L_{\phi_{1}, \cdots, \phi_{n}} u \equiv p_{n}\left[p_{n-1}\left(\cdots\left(p_{0} u\right)^{\prime} \cdots\right)^{\prime}\right]^{\prime}
$$


is "the" global C.F. of $L_{\phi_{1}, \cdots, \phi_{n}}$ of type (I) at $x_{0}$ and it turns out to be of type (II) at $T$.

(iv) The special fundamental system of solutions to $L_{\phi_{1}, \cdots, \phi_{n}} u=0$ defined by

$$
P_{0}(x):=\frac{1}{p_{0}(x)} ; \quad P_{i}(x):=\frac{1}{p_{0}(x)} \int_{T}^{x} \frac{1}{p_{1}} \cdots \int_{T}^{t_{i-1}} \frac{1}{p_{i}}, \quad 1 \leq i \leq n-1
$$

satisfies the asymptotic relations:

$$
\begin{cases}P_{0}(x) \gg P_{1}(x) \gg \ldots \gg P_{n-2}(x) \gg P_{n-1}(x), & x \rightarrow T^{+} \\ P_{n-1}(x) \gg P_{n-2}(x) \gg \ldots \gg P_{1}(x) \gg P_{0}(x), & x \rightarrow x_{0}^{-} .\end{cases}
$$

Relations (2.38) uniquely determine the fundamental system $\left(P_{0}, \cdots, P_{n-1}\right)$ up to multiplicative constants. (In the terminology used by the author [2] [3] the $n$-tuple $\left(P_{0}, \cdots, P_{n-1}\right)$ is a "mixed hierarchical system" on ]$T, x_{0}$ [ whereas Levin [5] (p. 80) would call it a "doubly hierarchical system" because he uses different arrangements for asymptotic scales at the left or right endpoints [5] (p. 59). If the $\phi_{i}$ 's are strictly positive then the same is true for all the Wronskians in (2.33) hence the absolute values are redundant; in this case it is the inverted $n$-tuple $\left(\phi_{n}, \cdots, \phi_{1}\right)$ which forms an extended complete Chebyshev system on $\left[T, x_{0}[\right.$.

The construction of the two above factorizations starting from the given expressions of the coefficients $q_{i}$ or $p_{i}$ is the classical procedure by Pólya [12]. Notice that the functions $p_{i}$ 's in (2.33), which are unique (constant factors apart) by a mentioned result by Trench, may be recovered from many different asymptotic scales and not just from one! The main feature of the above proposition is that we can express all the properties of our basic operator (at least those needed in our theory) in terms of the a-priori given Chebyshev asymptotic scale. The use of absolute values in the definitions of the $q_{i}$ 's and $p_{i}$ 's has the advantage of avoiding their use in the everywhere-present integral representations; and we must use them in at least one of the definitions as the two sets of Wronskians cannot have one and the same sign.

\section{Applying Differential Operators to Asymptotic Scales}

In the elementary case of Taylor's formula, the simple condition

$$
\exists f^{(n)}\left(x_{0}\right)
$$

is not a mere sufficient condition for the validity of the asymptotic expansion

$$
f(x)=\sum_{i=0}^{n} a_{i}\left(x-x_{0}\right)^{i}+o\left(\left(x-x_{0}\right)^{n}\right) \equiv T_{n}(x)+o\left(\left(x-x_{0}\right)^{n}\right), \quad x \rightarrow x_{o},
$$

it in fact characterizes the set of the $n$ asymptotic expansions

$$
f^{(k)}(x)=\sum_{i=0}^{n-k} T_{n}^{(k)}(x)+o\left(\left(x-x_{0}\right)^{n-k}\right), \quad x \rightarrow x_{0}, 0 \leq k \leq n-1,
$$

which is formed by (3.2) together with the relations obtained by formal differentiation $1,2, \cdots, n-1$ times. In this case, we have the known formulas for the coeffficients:

$$
a_{i}=f^{(i)}\left(x_{0}\right) / i !, \quad 0 \leq i \leq n .
$$

If we strenghten condition (3.1) by assuming

$$
f \in A C^{n}\left(I_{x_{0}}\right), \quad I_{x_{0}}: \text { a neighborhood of } x_{o},
$$

we also have the representation

$$
f^{(n)}(x)=f^{(n)}\left(x_{0}\right)+\int_{x_{0}}^{x} f^{(n+1)}(t) \mathrm{d} t
$$

which, besides implying the validity of (3.3) for $k=n$ as well, gives rise to the integral representation formu- 
las of all the remainders in (3.3). A similar situation occurs in the factorizational theory of polynomial asymptotic expansions at $+\infty$, [1], where the standard operator of differentiation $D:=\mathrm{d} / \mathrm{d} x$ happens to be formally applicable $n$ times to the expansion

$$
f(x)=a_{n} x^{n}+\cdots+a_{1} x+a_{0}+o(1), \quad x \rightarrow+\infty,
$$

in two quite different senses and under suitable integrability conditions. But in the analogous theory for expansions in arbitrary real powers

$$
f(x)=a_{1} x^{\alpha_{1}}+\ldots+a_{n} x^{\alpha_{n}}+o\left(x^{\alpha_{n}}\right), \quad x \rightarrow+\infty,\left(\alpha_{1}>\cdots>\alpha_{n}\right)
$$

developed in [3], it turns out that the most natural operators on which to build a satisfying theory are those linked to the C.F.'s of the differential operator in (2.20) with $\phi_{i}(x):=x^{\alpha_{i}}$ and not the operators $D^{k}$ though, in this special instance, the set of the formally-differentiated expansions may be equivalently expressed by expansions involving the standard derivatives. In the present general context Levin's theorem on hierarchies suggests that the $k$ th-order linear operators

$$
\mathcal{L}_{\phi_{i_{1}}, \cdots, \phi_{i_{k}}} u:=W\left(\phi_{i_{1}}, \cdots, \phi_{i_{k}}, u\right), \quad 1 \leq i_{1}<i_{2}<\cdots<i_{k} \leq n ; \quad 1 \leq k \leq n-1
$$

are likely to be formally applicable to an expansion (1.1) because they preserve the hierarchy (2.17) after suppressing the identically-zero terms, which means that they transform an asymptotic expansion with a zero remainder

$$
f(x)=a_{1} \phi_{1}(x)+\cdots+a_{n} \phi_{n}(x)
$$

into a similar expansion, namely:

$$
\mathcal{L}_{\phi_{1}, \cdots, \phi_{i_{k}}} f=\sum_{i \neq i_{j} \forall j}^{i=1, \cdots, n} a_{i} W\left(\phi_{i_{1}}, \cdots, \phi_{i_{k}}, \phi_{i}\right)
$$

For instance, we have the identity:

$$
\mathcal{L}_{\phi_{k}} f=a_{1} W\left(\phi_{k}, \phi_{1}\right)+\cdots+a_{k-1} W\left(\phi_{k}, \phi_{k-1}\right)+a_{k+1} W\left(\phi_{k}, \phi_{k+1}\right)+\cdots+a_{n} W\left(\phi_{k}, \phi_{n}\right)
$$

wherein

$$
W\left(\phi_{k}, \phi_{1}\right) \gg W\left(\phi_{k}, \phi_{2}\right) \gg \cdots \gg W\left(\phi_{k}, \phi_{k-1}\right) \gg W\left(\phi_{k}, \phi_{k+1}\right) \gg \cdots \gg W\left(\phi_{k}, \phi_{n}\right), \quad x \rightarrow x_{0}^{-}
$$

for each fixed $k, 1 \leq k \leq n-1, n \geq 3$ (For $n=2$ the chain (3.13) has only one term).

Now in the Wronskians (3.9) a permutation of $\left(\phi_{i_{1}}, \cdots, \phi_{i_{k}}\right)$ seems to be immaterial (a sign apart) but the object of our study, in a general formulation, involves a sequence of "nested" operators:

$$
\mathcal{L}_{\phi_{11}}, \mathcal{L}_{\phi_{i_{1}}, \phi_{i_{2}}}, \cdots, \mathcal{L}_{\phi_{i_{1}}, \phi_{i_{2}}, \cdots, \phi_{i_{k}}}
$$

where "nested" refers to the inclusions of their kernels and the problem consists in finding sufficient, and possibly necessary, conditions for the validity of the set of asymptotic relations

$$
\left\{\begin{array}{l}
f(x)=\sum_{i=1}^{n} a_{i} \phi_{i}(x)+o\left(\phi_{n}(x)\right) ; \\
\mathcal{L}_{\phi_{1}}[f(x)]=\sum_{i \neq i_{1}}^{j=1, \cdots, n} a_{i} W\left(\phi_{i_{1}}, \phi_{i} ; x\right)+o\left(\psi_{1}(x)\right) ; \\
\quad \ldots \\
\mathcal{L}_{\phi_{\phi_{1}}, \cdots, \phi_{i_{k}}}[f(x)]=\sum_{i \neq i_{j} \forall j}^{j=1, \cdots, n} a_{i} W\left(\phi_{i_{1}}, \cdots, \phi_{i_{k}}, \phi_{i} ; x\right)+o\left(\psi_{k}(x)\right) .
\end{array}\right.
$$


with proper choices of the $\psi_{i}$ 's. Once a subset $\left(\phi_{i_{1}}, \cdots, \phi_{i_{k}}\right)$ has been fixed there is no a-priori reason to prefer one permutation of the $\phi_{i}$ 's to another but it turns out that each ordered $k$-tuple $\left(\phi_{i_{1}}, \cdots, \phi_{i_{k}}\right)$ is linked to a special factorization of $\mathcal{L}_{\phi_{1}, \cdots, \phi_{i k}}$, possibly valid on a neighborhood of $x_{0}$ smaller than $\left[T, x_{0}\right.$ [ and calculations (to obtain meaningful results) can be successfully carried out only under proper integrability assumptions on the coefficients of the factorization, hence the order of the $\phi_{i}$ 's is not immaterial. A generic factorization of $\mathcal{L}_{\phi_{1}, \cdots, \phi_{n}}$, say (2.3), assumed valid on $\left[T, x_{0}[\right.$, involves the differential operators

$$
r_{0}(x) u ; \quad r_{1}(x)\left(r_{0}(x) u\right)^{\prime} ; \quad r_{2}(x)\left[r_{1}(x)\left(r_{0}(x) u\right)^{\prime}\right]^{\prime} ; \ldots
$$

which we label as "weighted derivatives of orders $0,1,2$ etc. with respect to the weights $\left(r_{0}, r_{1}, \cdots, r_{n}\right)$ ". Operators (3.16) are not always linked to operators of the type in (3.9) nor they preserve the hierarchy of the $\phi_{i}$ 's but the two C.F.'s highlighted in Proposition 2.1 yield two sequences of differential operators of orders $0,1,2, \cdots, n-1$ which are strictly related to operators in (3.9) and preserve the hierarchy; these operators were the core of the asymptotic theory in the case of real-power expansions [3] [13] hence they deserve a special attention and, as a matter of fact, the most meaningful results of our theory are based on them.

Referring to the factorization of type (I) in (2.36), with the $p_{i}$ 's in (2.33), we define the differential operators acting on $A C^{n-1}\left[T, x_{0}[\right.$ :

$$
L_{0} u:=p_{0}(x) u ; \quad L_{k} u:=p_{k}\left[p_{k-1}\left(\cdots\left(p_{0} u\right)^{\prime} \cdots\right)^{\prime}\right]^{\prime}, 1 \leq k \leq n ; \quad L_{n} u \equiv L_{\phi_{1}, \cdots, \phi_{n}} u
$$

which satisfy the recursive formula

$$
L_{k} u:=p_{k}(x)\left(L_{k-1} u\right)^{\prime}, \quad 1 \leq k \leq n .
$$

And referring to the factorization of type (II) in (2.29), with the $q_{i}$ 's in (2.25), we define the differential operators acting on $A C^{n-1}\left[T, x_{0}[\right.$ :

$$
M_{0} u:=q_{0}(x) u ; \quad M_{k} u:=q_{k}(x)\left[q_{k-1}(x)\left(\cdots\left(q_{0}(x) u\right)^{\prime} \cdots\right)^{\prime}\right]^{\prime}, 1 \leq k \leq n ; \quad M_{n} u \equiv L_{\phi_{1}, \cdots, \phi_{n}} u
$$

which satisfy the recursive formula

$$
M_{k} u:=p_{k}(x)\left(M_{k-1} u\right)^{\prime}, \quad 1 \leq k \leq n .
$$

Now representations (2.30) and (2.36) imply that:

$$
\text { ker } L_{k}=\operatorname{span}\left(\phi_{n}, \phi_{n-1}, \cdots, \phi_{n-k+1}\right) ; \quad \operatorname{ker} M_{k}=\operatorname{span}\left(\phi_{1}, \cdots, \phi_{k}\right), \quad 1 \leq k \leq n-1 \text {; }
$$

hence, there exists never-vanishing functions $\widetilde{p_{k}}, \widetilde{q_{k}}$ such that:

$$
L_{k} u=\widetilde{p_{k}} \cdot W\left(\phi_{n}, \phi_{n-1}, \cdots, \phi_{n-k+1}, u\right) ; \quad M_{k} u=\widetilde{q_{k}} \cdot W\left(\phi_{1}, \cdots, \phi_{k}, u\right), \quad 1 \leq k \leq n-1 .
$$

It follows that $L_{k}$ and $M_{k}$ preserve the hierarchy (2.17), namely we have the following asymptotic scales

$$
\begin{aligned}
& L_{k}\left[\phi_{1}(x)\right] \gg L_{k}\left[\phi_{2}(x)\right] \gg \cdots \gg L_{k}\left[\phi_{n-k}(x)\right], \quad x \rightarrow x_{o}^{-} ; \\
& M_{k}\left[\phi_{k+1}(x)\right] \gg M_{k}\left[\phi_{k+2}(x)\right] \gg \cdots \gg M_{k}\left[\phi_{n}(x)\right], \quad x \rightarrow x_{o}^{-} ;
\end{aligned}
$$

for each fixed $k, 0 \leq k \leq n-2$. For $k=0$ they respectively reduce to

$$
p_{0}(x) \phi_{1}(x) \gg p_{0}(x) \phi_{2}(x) \gg \cdots \gg p_{0}(x) \phi_{n}(x), \quad x \rightarrow x_{o}^{-} ;
$$




$$
q_{0}(x) \phi_{1}(x) \gg q_{0}(x) \phi_{2}(x) \gg \cdots \gg q_{0}(x) \phi_{n}(x), \quad x \rightarrow x_{o}^{-} ;
$$

both equivalent to (2.17). Hence, applying each $n$-tuple of operators $L_{k}$ and $M_{k}, 0 \leq k \leq n-1$, to (3.10) yields again asymptotic expansions with zero remainders and in this sense we may say that "the asymptotic expansion (3.10) is formally differentiable $(n-1)$ times with respect to the $n$-tuples of weights $\left(p_{0}, \cdots, p_{n-1}\right)$ and $\left(q_{0}, \cdots, q_{n-1}\right)$ " neglecting the $n$ th-order weighted derivatives which yield identically-zero expressions. The above discussion leads to the following

Conjecture. For each chosen C.F. of $L_{\phi_{1}, \cdots, \phi_{n}}$ of type either (I) or (II) at $x_{0}$,

$$
L_{\phi_{1}, \cdots, \phi_{n}} u \equiv r_{n}\left[r_{n-1}\left(\cdots\left(r_{0} u\right)^{\prime} \cdots\right)^{\prime}\right]^{\prime} \quad \forall u \in A C^{n-1}\left[T, x_{0}[\text {, }\right.
$$

there exists a linear subspace $\mathcal{D} \in A C^{n-1}\left[T, x_{0}[\right.$, such that:

(i) $\mathcal{D} \underset{\neq}{\supset} \operatorname{span}\left(\phi_{1}, \cdots, \phi_{n}\right)$

(ii) each $f \in \mathcal{D}$ has an asymptotic expansion of type (1.1) which is formally differentiable $(n-1)$ times with respect to the $n$-tuples of weights $\left(r_{0}, r_{1}, \cdots, r_{n-1}\right)$.

The problem consists in finding out analytic conditions characterizing the elements of $\mathcal{D}$ for a C.F. of type (I) or (II) separately. The foregoing approach suggests a smallness condition involving the quantity $L_{\phi_{1}, \cdots, \phi_{n}}[f(x)]$ which is $\equiv 0$ whenever the remainder in the expansion is.

There is another kind of considerations suggesting a special role of C.F.'s of type (II). If we wish to investigate the possible expressions of the coefficients of an asymptotic expansion alternatively to the recurrent formulas (1.3), so generalizing (3.4), it is clear from the study of polynomial expansions in [1] that the C.F. of type (I) is of no use to this end whereas the right approach is via a C.F. of type (II) by establishing a link between the coefficients of (3.10) and the limits of the weighted derivatives.

Proposition 3.1 (The coefficients of an asymptotic expansion with zero remainder). Referring to the T.A.S. in Proposition 2.4 and to the special factorization (2.29) the following facts hold true for the differential operators $M_{k}$ in (3.19):

(I) The $M_{k}$ 's satisfy the following relations:

$$
\begin{gathered}
M_{k}\left[\phi_{i}(x)\right]=0, \quad 1 \leq i \leq k ; \quad M_{k}\left[\phi_{k+1}(x)\right] \equiv \epsilon_{k}=\text { constant }= \pm 1, \quad 1 \leq k \leq n-1 \\
\left\{\begin{array}{l}
M_{k}\left[\phi_{h}(x)\right]=\epsilon_{h, k} \cdot \int_{x}^{x_{0}} \frac{1}{q_{k+1}} \cdots \int \frac{1}{q_{h-1}}=o(1), \quad x \rightarrow x_{0}^{-} ; \\
\epsilon_{h, k}=\text { constant }= \pm 1, \quad 1 \leq k \leq h-2, \quad h \leq n ;
\end{array}\right. \\
M_{k} u \equiv \epsilon_{k} \frac{W\left(\phi_{1}, \cdots, \phi_{k}, u\right)}{W\left(\phi_{1}, \cdots, \phi_{k}, \phi_{k+1}\right)}, \quad 1 \leq k \leq n-1 .
\end{gathered}
$$

(II) For a fixed $k, 1 \leq k \leq n$, we have the logical equivalence:

$$
M_{k-1}[f(x)] \equiv \epsilon_{k-1} \cdot a_{k}=\text { constant on some interval } J
$$

iff

$$
f(x)=a_{1} \phi_{1}(x)+\ldots+a_{k} \phi_{k}(x) \text { on } J \text { for some constants } a_{i}
$$

$a_{k}$ being the same as in (3.31) and $\epsilon_{k-1}$ as in (3.28).

If (3.31)-(3.32) hold true on a left neighborhood of $x_{0}$ then the following limits exist as finite numbers and

$$
\epsilon_{h-1} \cdot a_{h}=\lim _{x \rightarrow x_{0}^{-}} M_{h-1}[f(x)], \quad 1 \leq h \leq k
$$

where, for $h=k$ (3.33) is the identity (3.31). 
(III) In the special case where all the Wronskians in (2.18) are strictly positive then the constants in (3.28)-(3.29) have the values:

$$
\epsilon_{k}=1, \quad \epsilon_{h, k}=(-1)^{h+k+1} .
$$

We stress that the equivalence " $(3.31) \Leftrightarrow(3.32)$ ” is an algebraic fact based on (3.28)-(3.29) whereas the inference "(3.31)-(3.32) $\Rightarrow$ (3.33)" is an asymptotic property whose validity requires that $\left(\phi_{1}, \cdots, \phi_{k}\right)$ be an asymptotic scale at $x_{0}$ and that the operators $M_{k}$ be defined as specified. The above proposition suggests the following

Conjecture. If all the limits in (3.33) exist as finite numbers for some function $f$ sufficiently regular on a left deleted neighborhood of $x_{0}$ then an asymptotic expansion

$$
f(x)=a_{1} \phi_{1}(x)+\ldots+a_{k} \phi_{k}(x)+o\left(\phi_{k}(x)\right), \quad x \rightarrow x_{o}^{-},
$$

holds true matched to other expansions obtained by formal applications of the operators $M_{1}, \cdots, M_{k-1}$. Moreover, it is worth investigating if the validity of the sole last relation in (3.33), i.e. for $h=k$, implies the validity of the other relations.

Our study gives complete answers to the above Conjectures and the main results are reported in the next sections.

\section{The First Factorizational Approach}

We start from the "unique" C.F. of our operator $L_{\phi_{1}, \cdots, \phi_{n}}$ on the interval $\left[T, x_{0}\left[\right.\right.$ of type (I) at $x_{0}$, i.e. identity (2.36) with conditions (2.35) and the $p_{i}$ 's satisfying the same conditions as do the $q_{i}$ 's in (2.26). In this approach, the appropriate differential operators are the $L_{k}$ 's defined in (3.17) which satisfy:

$$
L_{k}\left[\phi_{n-i}(x)\right] \equiv \begin{cases}b_{n-k}, & 0 \leq i=k \leq n-1, \\ 0 & 0 \leq i<k .\end{cases}
$$

with suitable non-zero constants $b_{i}$ 's, and we have the asymptotic scales as $x \rightarrow x_{0}^{-}$:

$$
\left\{\begin{array}{l}
L_{0}\left[\phi_{1}(x)\right] \gg L_{0}\left[\phi_{2}(x)\right] \gg \cdots \gg L_{0}\left[\left[\phi_{n}(x)\right] ;\right. \\
L_{1}\left[\phi_{1}(x)\right] \gg L_{1}\left[\phi_{2}(x)\right] \gg \cdots \gg L_{1}\left[\phi_{n-1}(x)\right] ; \\
L_{2}\left[\phi_{1}(x)\right] \gg L_{2}\left[\phi_{2}(x)\right] \gg \cdots \gg L_{2}\left[\phi_{n-2}(x)\right] ; \\
\quad \cdots \\
L_{n-2}\left[\phi_{1}(x)\right] \gg L_{n-2}\left[\phi_{2}(x)\right] .
\end{array}\right.
$$

Moreover any function $f \in A C^{n-1}\left[T, x_{0}[\right.$ admits of a representation of type:

$$
f(x)=c_{1} \phi_{1}(x)+\cdots+c_{n} \phi_{n}(x)+\frac{1}{p_{0}(x)} \int_{T}^{x} \frac{1}{p_{1}} \cdots \int_{T}^{t_{n-2}} \frac{1}{p_{n-1}} \int_{T}^{t_{n-1}} \frac{L_{\phi_{1}, \cdots, \phi_{n}}[f(t)]}{p_{n}(t)} \mathrm{d} t, \quad x \in\left[T, x_{0}[\right.
$$

with suitable constants $c_{i}$. And applying the operators $L_{k}$ to (4.3), we get the following representations of the weighted derivatives with respect to the weight functions $\left(p_{1}, \cdots, p_{n-1}\right)$ :

$$
\left\{\begin{array}{l}
L_{k}[f(x)]=c_{1} L_{k}\left[\phi_{1}(x)\right]+\cdots+c_{n-k} L_{k}\left[\phi_{n-k}(x)\right]+\int_{T}^{x} \frac{\mathrm{d} t_{k+1}}{p_{k+1}\left(t_{k+1}\right)} \cdots \int_{T}^{t_{n-2}} \frac{\mathrm{d} t_{n-1}}{p_{n-1}\left(t_{n-1}\right)} \int_{T}^{t_{n-1}} \frac{L_{\phi_{1}, \cdots, \phi_{n}}[f(t)]}{p_{n}(t)} \mathrm{d} t, \\
1 \leq k \leq n-2 ; \\
L_{n-1}[f(x)]=c_{1} b_{1}+\int_{T}^{x} \frac{L_{\phi_{1}, \cdots, \phi_{n}}[f(t)]}{p_{n}(t)} \mathrm{d} t, \quad b_{1} \text { as in (4.1). }
\end{array}\right.
$$

Here is one of the main results obtainable by this approach. 
Theorem 4.1 (Asymptotic expansions formally differentiable according to the C.F. of type (I)). For $f \in A C^{n-1}\left[T, x_{0}[\right.$ the following are equivalent properties:

(i) The set of asymptotic expansions as $x \rightarrow x_{0}^{-}$for suitable constants $a_{1}, \cdots, a_{n}$ :

$$
\left\{\begin{array}{l}
f(x)=a_{1} \phi_{1}(x)+\cdots+a_{n} \phi_{n}(x)+o\left(\phi_{n}(x)\right), \\
L_{k}[f(x)]=a_{1} L_{k}\left[\phi_{1}(x)\right]+\cdots+a_{n-k} \underbrace{L_{k}\left[\phi_{n-k}(x)\right]}_{\text {constant }}+o(1), \quad 1 \leq k \leq n-1,
\end{array}\right.
$$

where the last term in each expansion is lost in the successive expansion.

(ii) The iterated improper integral

$$
\int_{T}^{x_{0}} \frac{1}{p_{1}} \cdots \int_{t_{n-2}}^{x_{0}} \frac{1}{p_{n-1}} \int_{t_{n-1}}^{x_{0}} \frac{L_{\phi_{1}, \cdots, \phi_{n}}[f(t)]}{p_{n}(t)} \mathrm{d} t \text { converges }
$$

(iii) There exist $n$ real numbers $a_{1}, \cdots, a_{n}$ and a function $\Phi_{n}$ Lebesgue-summable on $\left[T, x_{0}[\right.$ such that

$$
\left.f(x)=a_{1} \phi_{1}(x)+\cdots+a_{n} \phi_{n}(x)\right)+\frac{(-1)^{n}}{p_{0}(x)} \int_{x}^{x_{0}} \frac{1}{p_{1}} \int_{t_{1}}^{x_{0}} \frac{1}{p_{2}} \cdots \int_{t_{n-2}}^{x_{0}} \frac{1}{p_{n-1}} \int_{t_{n-1}}^{x_{0}} \Phi_{n} \mathrm{~d} t, \quad x \in\left[T, x_{0}[.\right.
$$

If this is the case $\Phi_{n}$ is determined up to a set of measure zero and

$$
\Phi_{n}(x)=\frac{1}{p_{n}(x)} L_{\phi_{1}, \cdots, \phi_{n}}[f(x)] \text { a.e. on }\left[T, x_{0}[\text {. }\right.
$$

The phenomenon appearing in (4.5) is intrinsic in the theory; it occurs even in the seemingly elementary case of real-power expansions, [3] (Thm. 4.2-(ii), p. 181, and formula (7.2), p. 195), where the asymptotic scale enjoys the most favourable algebraic properties. This type of formal differentiation of an asymptotic expansion does not frequently occur in the literature though the results in this section show that it is one of the possible natural situations. An instance (not inserted in a general theory) is to be found in a paper by Schoenberg [14] (Thm. 3, p. 258) and refers to the asymptotic expansion

$$
f(x)=a_{1} x^{-1}+a_{2} x^{-2}+\cdots+a_{n} x^{-n}+O\left(x^{-n-1}\right), \quad x \rightarrow+\infty .
$$

Starting from an "incomplete asymptotic expansion"

$$
f(x)=a_{i} \phi_{1}(x)+\cdots+a_{n} \phi_{i}(x)+o\left(\phi_{i}(x)\right), \quad 1 \leq i \leq n-1,
$$

our study would characterize a set of more involved expansions not reported here.

\section{The Second Factorizational Approach and Numerical Estimates of the Remainder}

Now, we face our problem starting from a C.F. of type (II) at $x_{0}$. Referring to Proposition 2.4 the most natural choice is the special C.F. of $L_{\phi_{1}, \cdots, \phi_{n}}$ in (2.29), with the $q_{i}$ 's in (2.25) and satisfying conditions (2.26). According to the Conjectures in $\S 3$ we shall characterize a set of asymptotic expansions, involving the operators $M_{k}$ defined in (3.19) and described in Proposition 3.1, wherein each coefficient of the first expansion may be found by an independent limiting process instead of the recursive formulas (1.3), and the existence of the sole last coefficient implies the existence of all the preceding coefficients.

In this new context, a representation of the following type is appropriate for any function $f \in A C^{n-1}\left[T, x_{0}[\right.$

$$
f(x)=c_{1} \phi_{1}(x)+\cdots+c_{n} \phi_{n}(x)+\frac{1}{q_{0}(x)} \int_{T}^{x} \frac{1}{q_{1}} \cdots \int_{T}^{t_{n-2}} \frac{1}{q_{n-1}} \int_{T}^{t_{n-1}} \frac{L_{\phi_{1}, \cdots, \phi_{n}}[f(t)]}{q_{n}(t)} \mathrm{d} t, \quad x \in\left[T, x_{0}[,\right.
$$


with suitable constants $c_{i}$. Applying the operators $M_{k}$ to (5.1) we get the following representations of the weighted derivatives of $f$ with respect to the weight functions $\left(q_{0}, \cdots, q_{n}\right)$

$$
M_{k}[f(x)]=c_{k+1} M_{k}\left[\phi_{k+1}(x)\right]+\cdots+c_{n} M_{k}\left[\phi_{n}(x)\right]+\int_{T}^{x} \frac{\mathrm{d} t_{k+1}}{q_{k+1}\left(t_{k+1}\right)} \cdots \int_{T}^{t_{n-2}} \frac{\mathrm{d} t_{n-1}}{q_{n-1}\left(t_{n-1}\right)} \int_{T}^{t_{n-1}} \frac{L_{\phi_{1}, \cdots, \phi_{n}}[f(t)]}{q_{n}(t)} \mathrm{d} t,
$$

To simplify formulas and to leave no ambiguity about the signs of the involved quantities we assume in this section that the Wronskians in (2.18) are strictly positive.

Hence, by (3.34) $\epsilon_{k}=1$ and the last relation in (5.2) explicitly is

$$
M_{n-1}[f(x)]=c_{n}+\int_{T}^{x} \frac{L_{\phi_{1}, \cdots, \phi_{n}}[f(t)]}{q_{n}(t)} \mathrm{d} t, \quad x \in\left[T, x_{0}[.\right.
$$

Theorem 5.1 (Asymptotic expansions formally differentiable according to a C.F. of type (II)). Let our T.A.S. be such that all the Wronskians in (2.18) are strictly positive and let $f \in A C^{n-1}\left[T, x_{0}[\right.$.

(I) The following are equivalent properties:

(i) There exist $n$ real numbers $a_{1}, \cdots, a_{n}$ such that:

$$
\begin{gathered}
f(x)=a_{1} \phi_{1}(x)+\cdots+a_{n} \phi_{n}(x)+o\left(\phi_{n}(x)\right), \quad x \rightarrow x_{o}^{-} ; \\
M_{k}[f(x)]=a_{k+1} M_{k}\left[\phi_{k+1}(x)\right]+\cdots+a_{n} M_{k}\left[\phi_{n}(x)\right]+o\left(M_{k}\left[\phi_{n}(x)\right]\right), \quad x \rightarrow x_{0}^{-}, \quad 1 \leq k \leq n-1,
\end{gathered}
$$

where the first term in each expansion is lost in the successive expansion as in Taylor's formula. (The relation that would be obtained in (5.5) for $k=0$ differed from relation in (5.4) by the common factor $q_{0}(x)$.)

(ii) All the following limits exist as finite numbers:

$$
\lim _{x \rightarrow x_{0}^{-}} M_{k}[f(x)] \equiv a_{k+1}, \quad 0 \leq k \leq n-1,
$$

where the $a_{k}$ 's coincide with those in (5.4).

(iii) The single last limit in (5.6) exists as a finite number, i.e.

$$
\lim _{x \rightarrow x_{0}^{-}} M_{n-1}[f(x)] \equiv a_{n}
$$

and (5.7) is nothing but the relation in (5.5) for $k=n-1$ which reads $M_{n-1}[f(x)]=a_{n}+o(1), x \rightarrow x_{0}^{-}$

(iv) The improper integral

$$
\int_{T}^{\rightarrow x_{0}} \frac{L_{\phi_{1}, \cdots, \phi_{n}}[f(t)]}{q_{n}(t)} \mathrm{d} t \quad \text { converges }
$$

and automatically also the iterated improper integral

$$
\left.\int_{T}^{x_{0}} \frac{1}{q_{1}} \int^{x o} \frac{1}{q_{2}} \cdots \int^{x o} \frac{1}{q_{n-1}} \int^{x o} \frac{L_{\phi_{1}}, \cdots, \phi_{n}}{q_{n}(t)} \mathrm{d}(t)\right] \text { converges }
$$

(v) There exist $n$ real numbers $a_{1}, \cdots, a_{n}$ and a function $\Psi_{n}$ Lebesgue-summable on $\left[T, x_{0}[\right.$ such that

$$
f(x)=\sum_{i=1}^{n} a_{i} \phi_{i}(x)+\frac{(-1)^{n}}{q_{0}(x)} \int_{x}^{x_{0}} \frac{1}{q_{1}} \cdots \int_{t_{n-2}}^{x_{0}} \frac{1}{q_{n-1}} \int_{t_{n-1}}^{x_{0}} \Psi_{n}(t) \mathrm{d} t, \quad x \in\left[T, x_{0}[,\right.
$$

where we remind that, by (2.25), $1 / q_{0}(x)=\phi_{1}(x)$. In this case, $\Psi_{n}$ is determined up to a set of measure zero and 


$$
\Psi_{n}(x)=\frac{1}{q_{n}(x)} L_{\phi_{1}, \cdots, \phi_{n}}[f(x)] \text { a.e. on }\left[T, x_{0}[\text {. }\right.
$$

(II) Whenever properties in part (I) hold true we have integral representation formulas for the remainders

$$
R_{0}(x):=f(x)-\sum_{i=1}^{n} a_{i} \phi_{i}(x) ; R_{k}(x):=M_{k}[f(x)]-\sum_{i=1}^{n-k} a_{k+i} M_{k}\left[\phi_{k+i}(x)\right], \quad 1 \leq k \leq n-1,
$$

namely:

$$
\begin{aligned}
& R_{0}(x)=\frac{(-1)^{n}}{q_{0}(x)} \int_{x}^{x_{0}} \frac{1}{q_{1}} \cdots \int_{t_{n-2}}^{x_{0}} \frac{1}{q_{n-1}} \int_{t_{n-1}}^{x_{0}} \frac{L_{\phi_{1}, \cdots, \phi_{n}}[f(t)]}{q_{n}(t)} \mathrm{d} t ; \\
& R_{k}(x)=(-1)^{n+k} \int_{x}^{x_{0}} \frac{1}{q_{k+1}} \cdots \int_{t_{n-2}}^{x_{0}} \frac{1}{q_{n-1}} \int_{t_{n-1}}^{x_{0}} \frac{L_{\phi_{1}, \cdots, \phi_{n}}[f(t)]}{q_{n}(t)} \mathrm{d} t ;
\end{aligned}
$$

for $x \in\left[T, x_{0}\left[, 1 \leq k \leq n-1\right.\right.$. From (5.13), we get the following estimate of $R_{0}$ wherein the order of smallness with respect to $\phi_{n}$ is made more explicit than in Theorem 4.1 (formula in (2.30) for $i=n$ is used):

$$
\left|R_{0}(x)\right| \leq\left|\phi_{n}(x)\right| \cdot \sup _{t \geq x}\left|\int_{t}^{x_{0}} \frac{L_{\phi_{1}, \cdots, \phi_{n}}[f(\tau)]}{q_{n}(\tau)} \mathrm{d} \tau\right|, \quad x \in\left[T, x_{0}[.\right.
$$

Under the stronger hypothesis of absolute convergence for the improper integral we get:

$$
\left|R_{0}(x)\right| \leq\left|\phi_{n}(x)\right| \cdot \int_{x}^{x_{0}} \frac{\left|L_{\phi_{1}, \cdots, \phi_{n}}[f(t)]\right|}{\left|q_{n}(t)\right|} \mathrm{d} t, \quad x \in\left[T, x_{0}[.\right.
$$

Similar estimates can be obtained for the $R_{k}$ 's.

Remarks. 1. As noticed in [3] (Remark 1 after Thm. 4.1, pp. 179, 180) the remarkable inference " (iii) $\Rightarrow$ (ii)" is true for the special operator $M_{n-1}$ stemming out from a C.F. of type (II) at $x_{0}$ but not for any $(n-1)$ th-order differential operator originating from an arbitrary factorization of $L_{\phi_{1}, \cdots, \phi_{n}}$.

2. Condition (5.8) involves the sole coefficient $q_{n}$ which admits of the explicit expression in (2.25) in terms of $\phi_{1}, \cdots, \phi_{n}: q_{n}=W\left(\phi_{1}, \cdots, \phi_{n}\right) / W\left(\phi_{1}, \cdots, \phi_{n-1}\right)$ hence (5.8) can be rewritten as

$$
\int_{T}^{\rightarrow x_{0}} \frac{W\left(\phi_{1}(t), \cdots, \phi_{n-1}(t)\right)}{W\left(\phi_{1}(t), \cdots, \phi_{n}(t)\right)} L_{\phi_{1}, \cdots, \phi_{n}}[f(t)] \mathrm{d} t \text { converges }
$$

For $n=2$, the ratio inside the integral equals $\phi_{1} / W\left(\phi_{1}, \phi_{2}\right)$ and we reobtain the result in [2], condition (5.15), p. 265.

3. In Theorem 4.1, generally speaking, no such estimates as in (5.15)-(5.16) can be obtained due to the divergence of all the improper integrals in (4.6) if the innermost integral is factored out.

4. It has been proved in [1] for polynomial expansions, in [3] for real-power expansions, and in [2] for twoterm expansions that properties in Proposition 5.1 are stronger than those in Proposition 4.1; this does not seem an easy fact to prove or disprove for a general expansion if $n \geq 3$. In the next section we highlight two cases (important for applications) wherein the two types of formal differentiability are equivalent.

\section{Absolute Convergence and Solutions of Differential Inequalities}

The foregoing theory becomes particularly simple when the involved improper integrals are absolutely convergent and still more expressive for a function $f$ satisfying the $n$ h-order differential inequality:

$$
L_{\phi_{1}, \cdots, \phi_{n}}[f(x)] \geq 0 \text { a.e. on }\left[T, x_{0}[\right.
$$

If $W\left(\phi_{1}(x), \cdots, \phi_{i}(x)\right)>0$ on $\left[T, x_{0}[, 1 \leq i \leq n\right.$, this is a subclass of the so-called "generalized convex fun- 
ions with respect to the (extended complete Chebyshev) system $\left(\phi_{1}, \cdots, \phi_{n}\right)$ ") and we make this assumption, as in the preceding section, to simplify relations involving the operators $M_{k}$ and to state precise inequalities for the remainders. The nice result stated in the next theorem claims that: if such a function admits of an asymptotic expansion (1.1) then this expansion is automatically differentiable $(n-1)$ times (which is a special circumstance) in the senses of both relations (4.4) and (5.5).

Theorem 6.1. If all the Wronskians in (2.18) are strictly positive and if $f \in A C^{n-1}\left[T, x_{0}\right.$ [ satisfies (6.1) then the following are equivalent properties:

(i) There exist $(n-1)$ real numbers $a_{1}, \cdots, a_{n-1}$ such that:

$$
f(x)=a_{1} \phi_{1}(x)+\cdots+a_{n-1} \phi_{n-1}(x)+O\left(\phi_{n}(x)\right), \quad x \rightarrow x_{0}^{-}
$$

(ii) There exist $n$ real numbers $a_{1}, \cdots, a_{n}$ such that:

$$
f(x)=a_{1} \phi_{1}(x)+\cdots+a_{n-1} \phi_{n-1}(x)+a_{n} \phi_{n}(x)+o\left(\phi_{n}(x)\right), \quad x \rightarrow x_{0}^{-}
$$

(iii) The following set of asymptotic expansions holds true:

$$
L_{k}[f(x)]=a_{1} L_{k}\left[\phi_{1}(x)\right]+\cdots+a_{n-k} \underbrace{L_{k}\left[\phi_{n-k}(x)\right]}_{\text {constant }}+o(1), \quad x \rightarrow x_{0}^{-}, \quad 0 \leq k \leq n-1 ; \quad \text { see (4.4) }
$$

(iv) The following set of asymptotic expansions holds true:

$$
\begin{aligned}
& M_{k}[f(x)]=a_{k+1} M_{k}\left[\phi_{k+1}(x)\right]+\cdots+a_{n} M_{k}\left[\phi_{n}(x)\right]+o\left(M_{k}\left[\phi_{n}(x)\right]\right), \\
& x \rightarrow x_{0}^{-} ; \quad 0 \leq k \leq n-1 ; \text { see (5.5) }
\end{aligned}
$$

(v) The following integral condition is satisfied:

$$
\left.\int_{T}^{x_{0}} \frac{1}{p_{1}} \cdots \int_{t_{n-2}}^{x_{0}} \frac{1}{p_{n-1}} \int_{t_{n-1}}^{x_{0}} \frac{L_{\phi_{1}}, \cdots, \phi_{n}}{p_{n}(t)} \mathrm{d}(t)\right]<+\infty ; \text { see (4.5) }
$$

(vi) The following integral condition is satisfied:

$$
\int_{T}^{x_{0}} \frac{1}{q_{n}(t)} L_{\phi_{1}, \cdots, \phi_{n}}[f(t)] \mathrm{d} t<+\infty \text {, see (5.8) and (5.17) }
$$

To this list, we may obviously add the other properties in Theorem 5.1.

If this is the case, the remainder $R_{0}(x)$ in (6.3) admits of the two representations on $\left[T, x_{0}[\right.$ :

$$
\begin{aligned}
R_{0}(x) & =\frac{(-1)^{n}}{p_{0}(x)} \int_{x}^{x_{0}} \frac{1}{p_{1}} \cdots \int_{t_{n-2}}^{x_{0}} \frac{1}{p_{n-1}} \int_{t_{n-1}}^{x_{0}} \frac{L_{\phi_{1}, \cdots, \phi_{n}}[f(t)]}{p_{n}(t)} \mathrm{d} t \\
& =\frac{(-1)^{n}}{q_{0}(x)} \int_{x}^{x_{0}} \frac{1}{q_{1}} \cdots \int_{t_{n-2}}^{x_{0}} \frac{1}{q_{n-1}} \int_{t_{n-1}}^{x_{0}} \frac{L_{\phi_{1}, \cdots, \phi_{n}}[f(t)]}{q_{n}(t)} \mathrm{d} t,
\end{aligned}
$$

whence it follows that $(-1)^{n} R_{0}(x) \geq 0 \forall x \in\left[T, x_{0}[\right.$.

In addition to the equivalence (iii) $\Leftrightarrow$ (iv) stated in Theorem 6.1, there is another remarkable circumstance wherein the two types of formal differentiations are simultaneously admissible namely when the convergence of the pertinent improper integrals is absolute.

Theorem 6.2. For $f \in A C^{n-1}\left[T, x_{0}[\right.$ the following integral conditions are equivalent:

$$
\int_{T}^{x_{0}} \frac{1}{p_{1}} \cdots \int_{t_{n-2}}^{x_{0}} \frac{1}{p_{n-1}} \int_{t_{n-1}}^{x_{0}} \frac{1}{p_{n}(t)}\left|L_{\phi_{1}, \cdots, \phi_{n}}[f(t)]\right| \mathrm{d} t<+\infty ;
$$




$$
\begin{gathered}
\left\{\begin{array}{l}
\int_{T}^{x_{0}} \tilde{P}_{n}(t)\left|L_{\phi_{1}, \cdots, \phi_{n}}[f(t)]\right| \mathrm{d} t<+\infty, \quad \text { where } \\
\tilde{P}_{n}(t):=\frac{1}{p_{n}(t)} \int_{T}^{t} \frac{\mathrm{d} t_{n-1}}{p_{n-1}\left(t_{n-1}\right)} \cdots \int_{T}^{t_{3}} \frac{\mathrm{d} t_{2}}{p_{2}\left(t_{2}\right)} \int_{T}^{t_{2}} \frac{\mathrm{d} t_{1}}{p_{1}\left(t_{1}\right)} ; \\
\int_{T}^{x_{0}} \frac{L_{\phi_{1}, \cdots, \phi_{n}}[f(t)] \mid}{q_{n}(t)} \mathrm{d} t \equiv \int_{T}^{x_{0}}\left|\frac{W\left(\phi_{1}(t), \cdots, \phi_{n-1}(t)\right)}{W\left(\phi_{1}(t), \cdots, \phi_{n}(t)\right)} L_{\phi_{1}, \cdots, \phi_{n}}[f(t)]\right| \mathrm{d} t<+\infty .
\end{array}\right.
\end{gathered}
$$

Hence, each of these three conditions implies both sets of asymptotic expansions (4.4) and (5.5). (Here the signs of the Wronskians are immaterial.)

The equivalence between (6.9) and (6.10) easily follows from Fubini's theorem by interchanging the order of integrations in (6.9) whereas the equivalence between (6.10) and (6.11) is by no means an obvious fact. A proof may be obtained by showing a stronger result, namely the following asymptotic relation:

$$
\tilde{P}_{n}(x) \sim\left|\frac{W\left(\phi_{1}(t), \cdots, \phi_{n-1}(t)\right)}{W\left(\phi_{1}(t), \cdots, \phi_{n}(t)\right)}\right|, \quad x \rightarrow x_{o}^{-} .
$$

\section{Example: A Special Class of Chebyshev Asymptotic Scales}

The foregoing results are well illustrated by the special class of scales of the form:

$$
(\phi(x))^{\alpha_{1}} \gg \cdots \gg(\phi(x))^{\alpha_{n}}, \quad x \rightarrow x_{o}^{-},
$$

where

$$
\left\{\begin{array}{l}
\text { either } \lim _{x \rightarrow x_{0}^{-}} \phi(x)=+\infty \text { and } \alpha_{1}>\cdots>\alpha_{n}, \\
\text { or } \quad \lim _{x \rightarrow x_{0}^{-}} \phi(x)=0^{+} \text {and } \alpha_{1}<\cdots<\alpha_{n} .
\end{array}\right.
$$

We also assume:

$$
\phi \in A C^{n-1}\left[T, x_{0}\left[; \quad \phi ( x ) > 0 ; \quad \phi ^ { \prime } ( x ) \neq 0 \text { on } \left[T, x_{0}[.\right.\right.\right.
$$

Now, using a proper device it can be given an elementary proof of the formula:

$$
\begin{aligned}
& W\left((g(x))^{\alpha_{1}}, \cdots,(g(x))^{\alpha_{n}}\right)=V\left(\alpha_{1}, \cdots, \alpha_{n}\right) \cdot(g(x))^{\alpha_{1}+\cdots+\alpha_{n}-[n(n-1) / 2]} \cdot\left(g^{\prime}(x)\right)^{n(n-1) / 2}, \\
& x \in\left[T, x_{0}[, \quad n \geq 2,\right.
\end{aligned}
$$

where $V\left(\alpha_{1}, \cdots, \alpha_{n}\right)$ denotes the Vandermonde determinant of the $n$ distinct numbers $\alpha_{1}, \cdots, \alpha_{n}$, hence our assumptions imply the non-vanishingness of all the Wronskians involved in our theory and the scale (7.1) is a Chebyshev asymptotic scale on $\left[T, x_{0}\left[\right.\right.$. We denote by $\tilde{L}_{\phi}$ the differential operator associated, as in (2.20), to our scale.

Proposition 7.1. Under the above assumptions and notations:

(I) The "unique" C.F. of $\tilde{L}_{\phi}$ of type (I) at $x_{0}^{-}$, constant factors apart, is:

$$
\left.\left.\tilde{L}_{\phi} u \equiv \phi^{\alpha_{1}}\left(\frac{\phi^{\prime}}{\phi}\right)^{n-1} \cdot\left[\frac{\phi^{\alpha_{2}-\alpha_{1}+1}}{\phi^{\prime}}\left(\ldots\left(\frac{\phi^{\alpha_{n-1}-\alpha_{n-2}+1}}{\phi^{\prime}}\left(\frac{\phi^{\alpha_{n}-\alpha_{n-1}+1}}{\phi^{\prime}}\left(\frac{u}{\phi^{\alpha_{n}}}\right)^{\prime}\right)\right)^{\prime}\right) \ldots\right)\right]^{\prime}\right]
$$

which also gives the differential operators $L_{1}, \cdots, L_{n-1}$ defined in (3.17).

(II) A special C.F. of $\tilde{L}_{\phi}$ of type (II) at $x_{0}^{-}$, apart from the signs of the coefficients, is:

$$
\tilde{L}_{\phi} u \equiv \phi^{\alpha_{n}}\left(\frac{\phi^{\prime}}{\phi}\right)^{n-1} \cdot\left[\frac{\phi^{\alpha_{n-1}-\alpha_{n}+1}}{\phi^{\prime}}\left(\ldots\left(\frac{\phi^{\alpha_{2}-\alpha_{3}+1}}{\phi^{\prime}}\left(\frac{\phi^{\alpha_{1}-\alpha_{2}+1}}{\phi^{\prime}}\left(\frac{u}{\phi^{\alpha_{1}}}\right)^{\prime}\right)^{\prime}\right)^{\prime} \ldots\right)^{\prime}\right]^{\prime}
$$


which also gives the differential operators $M_{1}, \cdots, M_{n-1}$ defined in (3.19).

Identities (7.5)-(7.6) can be proved either using (7.4) and formulas in Proposition 2.4 or writing out the $n$ tuple (2.37) and checking that its span coincides with $\operatorname{ker} \tilde{L}_{\phi}$. Now, we can specialize Theorems 4.1, 5.1.

Proposition 7.2. (I) Referring to Theorem 4.1 we have the equivalence of the following three properties:

(i) The set of asymptotic expansions as $x \rightarrow x_{0}^{-}$for suitable constants $a_{1}, \cdots, a_{n}$

$$
\left\{\begin{array}{l}
f(x)=a_{1}(\phi(x))^{\alpha_{1}}+\cdots+a_{n}(\phi(x))^{\alpha_{n}}+o\left((\phi(x))^{\alpha_{n}}\right) ; \\
L_{k}[f(x)]=a_{1} L_{k}\left[(\phi(x))^{\alpha_{1}}\right]+\cdots+a_{n-k} \underbrace{L_{k}\left[(\phi(x))^{\alpha_{n-k}}\right]}_{\text {constant }}+o(1), \quad 1 \leq k \leq n-1 .
\end{array}\right.
$$

(ii) The improper integral

$$
\int_{T}^{x_{0}} \frac{\phi^{\prime}}{\phi^{\alpha_{n}-\alpha_{n-1}+1}} \int_{t_{1}}^{x_{0}} \frac{\phi^{\prime}}{\phi^{\alpha_{n-1}-\alpha_{n-2}+1}} \ldots \int_{t_{n-2}}^{x_{0}} \frac{\phi^{\prime}}{\phi^{\alpha_{2}-\alpha_{1}+1}} \int_{t_{n-1}}^{x_{0}} \frac{(\phi(t))^{n-1-\alpha_{1}}}{\left(\phi^{\prime}(t)\right)^{n-1}} \tilde{L}_{\phi}[f(t)] \mathrm{d} t \text { converges }
$$

(iii) For suitable constants $a_{1}, \cdots, a_{n}$ the following representation holds true on $\left[T, x_{0}[\right.$

$$
\begin{aligned}
f(x)= & a_{1}(\phi(x))^{\alpha_{1}}+\cdots+a_{n}(\phi(x))^{\alpha_{n}} \\
& +(-1)^{n}(\phi(x))^{\alpha_{n}} \int_{x}^{x_{0}} \frac{\phi^{\prime}}{\phi^{\alpha_{n}-\alpha_{n-1}+1}} \int_{t_{1}}^{x_{0}} \frac{\phi^{\prime}}{\phi^{\alpha_{n-1}-\alpha_{n-2}+1}} \cdots \int_{t_{n-2}}^{x_{0}} \frac{\phi^{\prime}}{\phi^{\alpha_{2}-\alpha_{1}+1}} \int_{t_{n-1}}^{x_{0}} \frac{(\phi(t))^{n-1-\alpha_{1}}}{\left(\phi^{\prime}(t)\right)^{n-1}} \tilde{L}_{\phi}[f(t)] \mathrm{d} t .
\end{aligned}
$$

(II) Referring to Theorem 5.1, we have the equivalence of the following three properties:

(iv) The set of asymptotic expansions as $x \rightarrow x_{0}^{-}$for suitable constants $a_{1}, \cdots, a_{n}$

$$
\left\{\begin{array}{l}
f(x)=a_{1}(\phi(x))^{\alpha_{1}}+\cdots+a_{n}(\phi(x))^{\alpha_{n}}+o\left((\phi(x))^{\alpha_{n}}\right), \\
M_{k}[f(x)]=a_{k+1} M_{k}\left[(\phi(x))^{\alpha_{k+1}}\right]+\cdots+a_{n} M_{k}\left[(\phi(x))^{\alpha_{n}}\right]+o\left(M_{k}\left[(\phi(x))^{\alpha_{n}}\right]\right), \quad 1 \leq k \leq n-1 .
\end{array}\right.
$$

(v) The improper integral

$$
\int_{T}^{\rightarrow x_{0}} \frac{(\phi(x))^{n-1-\alpha_{n}}}{\left(\phi^{\prime}(x)\right)^{n-1}} \tilde{L}_{\phi}[f(t)] \mathrm{d} t \text { converges }
$$

(vi) For suitable constants $a_{1}, \cdots, a_{n}$, the following representation holds true on $\left[T, x_{0}[\right.$

$$
\begin{aligned}
f(x)= & a_{1}(\phi(x))^{\alpha_{1}}+\ldots+a_{n}(\phi(x))^{\alpha_{n}} \\
& +(-1)^{n}(\phi(x))^{\alpha_{1}} \int_{x}^{x_{0}} \frac{\phi^{\prime}}{\phi^{\alpha_{1}-\alpha_{2}+1}} \int_{t_{1}}^{x_{0}} \frac{\phi^{\prime}}{\phi^{\alpha_{2}-\alpha_{3}+1}} \ldots \int_{t_{n-2}}^{x_{0}} \frac{\phi^{\prime}}{\phi^{\alpha_{n-1}-\alpha_{n}+1}} \int_{t_{n-1}}^{x_{0}} \frac{(\phi(x))^{n-1-\alpha_{n}}}{\left(\phi^{\prime}(x)\right)^{n-1}} \tilde{L}_{\phi}[f(t)] \mathrm{d} t .
\end{aligned}
$$

We visualize Proposition 7.2 for five remarkable choices of $\phi$ and $x_{0}=+\infty$ in a simplified form listing the forms assumed by condition (7.11) and the respective expansions they imply (without derivatives). The same symbol $\tilde{L}_{\phi}$ obviously has a different meaning in each case, being the operator of type (2.1) whose kernel is spanned by the asymptotic scale at hand.

Corollary 7.3. (I) $(\phi(x):=\log x)$. For any real numbers $\alpha_{1}>\alpha_{2}>\cdots>\alpha_{n}$

$$
\left\{\begin{array}{l}
\int^{+\infty}(\log t)^{n-1-\alpha_{n}} t^{n-1} \tilde{L}_{\phi}[f(t)] \mathrm{d} t \quad \text { convergent } \Rightarrow \\
f(x)=\sum_{i=1}^{n} a_{i}(\log x)^{\alpha_{i}}+o\left((\log x)^{\alpha_{n}}\right), \quad x \rightarrow+\infty
\end{array}\right.
$$


(II) $(\phi(x):=x)$. For any real numbers $\alpha_{1}>\alpha_{2}>\cdots>\alpha_{n}$

$$
\int^{+\infty} t^{n-1-\alpha_{n}} \tilde{L}_{\phi}[f(t)] \mathrm{d} t \text { convergent } \Rightarrow f(x)=\sum_{i=1}^{n} a_{i} x^{\alpha_{i}}+o\left(x^{\alpha_{n}}\right), \quad x \rightarrow+\infty
$$

see the theory developed in [3].

(III) $\left(\phi(x):=\exp \left[(\log x)^{\alpha}\right]\right)$. For any real numbers $c_{1}>c_{2}>\cdots>c_{n}$ and $\alpha>0, \alpha \neq 1$

$$
\begin{cases}\int^{+\infty} \exp \left[-c_{n}(\log t)^{\alpha}\right](\log t)^{(1-\alpha)(n-1)} t^{n-1} \tilde{L}_{\phi}[f(t)] \mathrm{d} t & \text { convergent } \Rightarrow \\ f(x)=\sum_{i=1}^{n} a_{i} \exp \left[c_{i}(\log x)^{\alpha}\right]+o\left(\exp \left[c_{n}(\log x)^{\alpha}\right]\right), & x \rightarrow+\infty .\end{cases}
$$

(IV) $\left(\phi(x):=\mathrm{e}^{x}\right)$. For any real numbers $c_{1}>c_{2}>\cdots>c_{n}$

$$
\int^{+\infty} \mathrm{e}^{-c_{n} t} \tilde{L}_{\phi}[f(t)] \mathrm{d} t \text { convergent } \Rightarrow f(x)=\sum_{i=1}^{n} a_{i} \mathrm{e}^{c_{i} x}+o\left(\mathrm{e}^{c_{n} x}\right), \quad x \rightarrow+\infty .
$$

(V) $\left(\phi(x):=\exp x^{\alpha}\right)$. For any real numbers $c_{1}>c_{2}>\cdots>c_{n}$ and $\alpha>0$

$$
\left\{\begin{array}{l}
\int^{+\infty} \exp \left[-c_{n} t^{\alpha}\right] t^{(1-\alpha)(n-1)} \tilde{L}_{\phi}[f(t)] \mathrm{d} t \quad \text { convergent } \Rightarrow \\
f(x)=\sum_{i=1}^{n} a_{i} \exp \left[c_{i} x^{\alpha}\right]+o\left(\exp \left[c_{n} x^{\alpha}\right]\right), \quad x \rightarrow+\infty .
\end{array}\right.
$$

\section{Acknowledgements}

The author thanks the referees for their helpful suggestions.

\section{References}

[1] Granata, A. (2007) Polynomial Asymptotic Expansions in the Real Domain: The Geometric, the Factorizational, and the Stabilization Approaches. Analysis Mathematica, 33, 161-198. http://dx.doi.org/10.1007/s10476-007-0301-0

[2] Granata, A. (2011) Analytic Theory of Finite Asymptotic Expansions in the Real Domain. Part I: Two-Term Expansions of Differentiable Functions. Analysis Mathematica, 37, 245-287. For an Enlarged Version with Corrected Misprints see arXiv:1405.6745v1 [math.CA]. http://dx.doi.org/10.1007/s10476-011-0402-7

[3] Granata, A. (2010) The Problem of Differentiating an Asymptotic Expansion in Real Powers. Part II: Factorizational Theory. Analysis Mathematica, 36, 173-218. http://dx.doi.org/10.1007/s10476-010-0301-3

[4] Ostrowski, A.M. (1976) Note on the Bernoulli-L’Hospital Rule. American Mathematical Monthly, 83, $239-242$. http://dx.doi.org/10.2307/2318210

[5] Levin, A.Yu. (1969) Non-Oscillation of Solutions of the Equation $x^{(n)}+p_{1}(t) x^{(n-1)}+\ldots+p_{n}(t) x=0$. Uspekhi Matematicheskikh Nauk, 24, 43-96; Russian Mathematical Surveys, 24, 43-99. http://dx.doi.org/10.1070/RM1969v024n02ABEH001342

[6] Coppel, W.A. (1971) Disconjugacy. Lecture Notes in Mathematics. Vol. 220, Springer-Verlag, Berlin.

[7] Trench, W.F. (1974) Canonical Forms and Principal Systems for General Disconjugate Equations. Transactions of the American Mathematical Society, 189, 139-327. http://dx.doi.org/10.1090/S0002-9947-1974-0330632-X

[8] Granata, A. (1980) Canonical Factorizations of Disconjugate Differential Operators. SIAM Journal on Mathematical Analysis, 11, 160-172. http://dx.doi.org/10.1137/0511014

[9] Granata, A. (1988) Canonical Factorizations of Disconjugate Differential Operators-Part II. SIAM Journal on Mathematical Analysis, 19, 1162-1173. http://dx.doi.org/10.1137/0519081

[10] Karlin, S. and Studden, W. (1966) Tchebycheff Systems: With Applications in Analysis and Statistics. Interscience, New York.

[11] Mazure, M.L. (2011) Quasi Extended Chebyshev Spaces and Weight Functions. Numerische Mathematik, 118, 79-108. http://dx.doi.org/10.1007/s00211-010-0312-9

[12] Pólya, G. (1922) On the Mean-Value Theorem Corresponding to a Given Linear Homogeneous Differential Equations. 
Transactions of the American Mathematical Society, 24, 312-324. http://dx.doi.org/10.2307/1988819

[13] Granata, A. (2010) The Problem of Differentiating an Asymptotic Expansion in Real Powers. Part I: Unsatisfactory or Partial Results by Classical Approaches. Analysis Mathematica, 36, 85-112. http://dx.doi.org/10.1007/s10476-010-0201-6

[14] Schoenberg, I.J. (1982) Two Applications of Approximate Differentiation Formulae: An Extremum Problem for Multiply Monotone Functions and the Differentiation of Asymptotic Expansions. Journal of Mathematical Analysis and Applications, 89, 251-261. http://dx.doi.org/10.1016/0022-247X(82)90101-9 
Scientific Research Publishing (SCIRP) is one of the largest Open Access journal publishers. It is currently publishing more than 200 open access, online, peer-reviewed journals covering a wide range of academic disciplines. SCIRP serves the worldwide academic communities and contributes to the progress and application of science with its publication.

Other selected journals from SCIRP are listed as below. Submit your manuscript to us via either submit@scirp.org or Online Submission Portal.
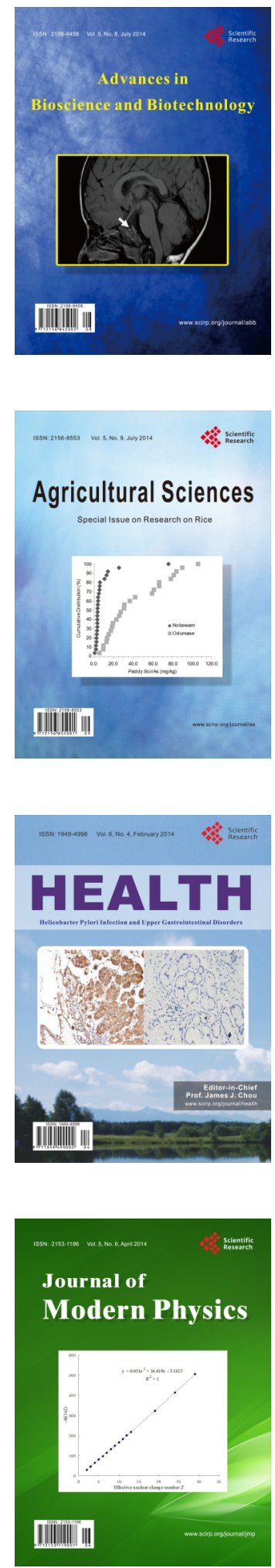
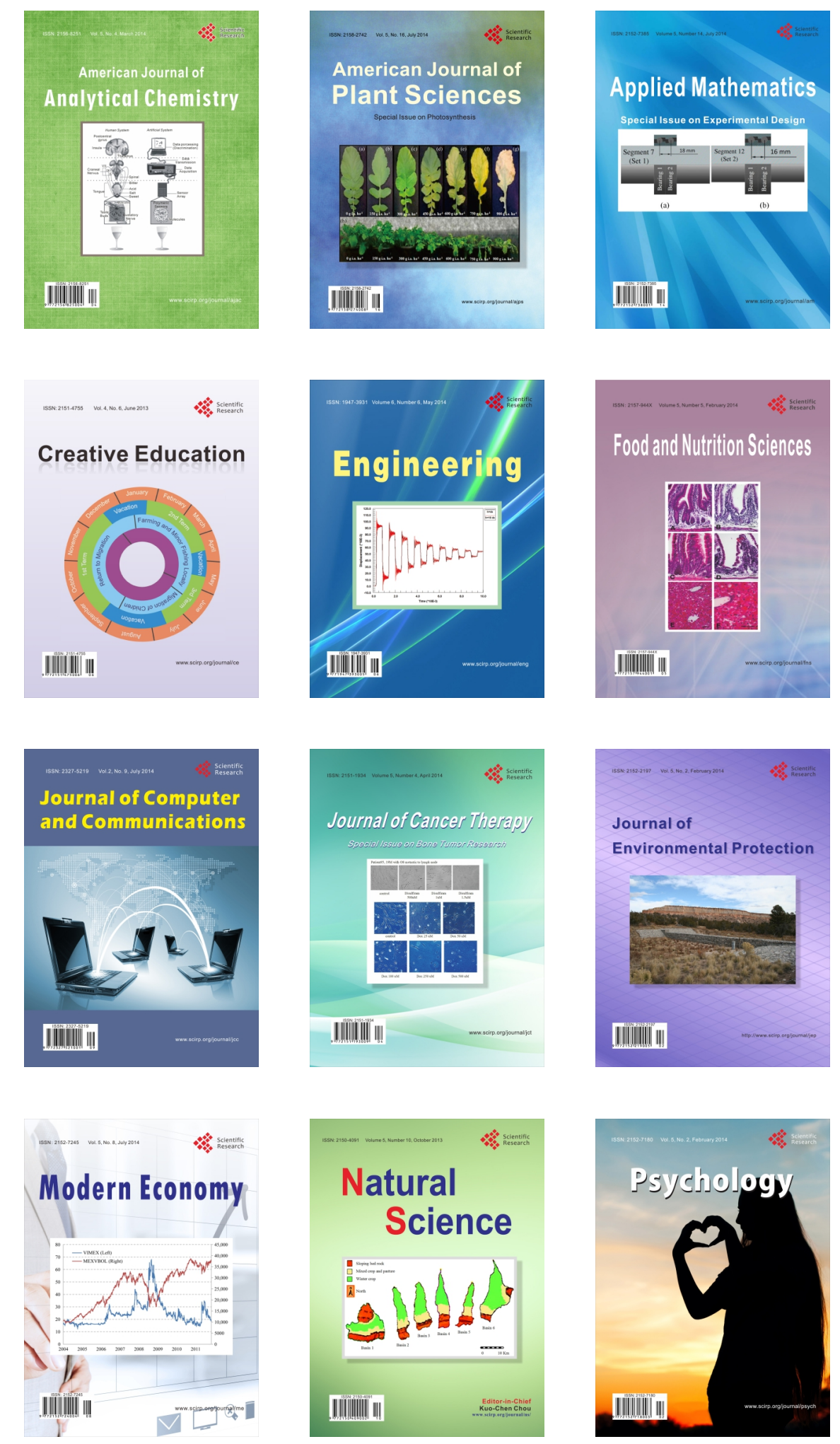